\title{
First-principles investigation of transient current in molecular devices by using complex absorbing potentials
}

\author{
Lei Zhang, Jian Chen, and Jian Wang* \\ Department of Physics and the Center of Theoretical and Computational Physics, The University of Hong Kong, Hong Kong, China
}

(Received 22 February 2013; revised manuscript received 4 April 2013; published 1 May 2013)

\begin{abstract}
Based on the nonequilibrium Green's function (NEGF) coupled with density function theory (DFT), namely, NEGF-DFT quantum transport theory, we propose an efficient formalism to calculate the transient current of molecular devices under a step-like pulse from first principles. By combining NEGF-DFT with the complex absorbing potential (CAP), the computational complexity of our formalism (NEGF-DFT-CAP) is proportional to $O(N)$ where $N$ is the number of time steps in the time-dependent transient current calculation. Compared with the state-of-the-art algorithm of first-principles time-dependent calculation that scales with at least $N^{2}$, this order $N$ technique drastically reduces the computational burden making it possible to tackle realistic molecular devices. We have presented a detailed discussion on how to implement this scheme numerically from first principles. To check the accuracy of our method, we carry out the benchmark calculation compared with NEGF-DFT formalism and they agree well with each other. As an application of this method, we investigate the transient current of a molecular device $\mathrm{Al}-\mathrm{C}_{3}-\mathrm{Al}$ from first principles.
\end{abstract}

DOI: 10.1103/PhysRevB.87.205401

PACS number(s): 71.15.Mb, 72.10.-d, 85.65.+h, 73.63.-b

\section{INTRODUCTION}

With the advance of nanofabrication techniques, nanodevices can be made using single atoms or molecules from the bottom-up approach, which leads to a new field of molecular electronics. ${ }^{1-7}$ Many experiments have been performed to measure quantum transport properties of molecular devices. ${ }^{2-7}$ At the same time, many research efforts have been made to understand these properties from first principles. ${ }^{8-11}$ At the present stage, the quantitative agreement between theoretical first-principles calculations and experimental results can be reached in DC transport when the coupling between molecules and contacts is strong. ${ }^{12-15}$ As for the weak coupling regime, people have made some progress in recent years. ${ }^{16-20}$ Besides the DC steady state problem, the question of how fast a molecular device can turn on and off is also an important issue, which has attracted a lot of research attention recently. ${ }^{21-31}$ In the design of a functional unit, it is important for us to know the short time response of molecular devices under switch on and off signal. Based on the dynamical information, one can study molecular charging and molecular discharging processes, which is helpful to characterize the behaviors of molecular devices. This kind of question can be answered by sending a step-like pulse from the electrodes and studying the dynamic response of molecular devices. For this problem, the exact solution of the transient current was obtained by Wingreen et al. ${ }^{21}$ in the wide-band limit using the nonequilibrium Green's function (NEGF) approach. Recently this solution has been extended to the regime of the finite bandwidth of electrodes. ${ }^{25}$ When applying this exact NEGF solution to molecular devices for the calculation of the transient current as a function of time, there is a huge computational cost due to the triple integral over energy. In addition, there are many quasipoles near the energy axis making the integration very difficult to converge. On the other hand, the theoretical prediction of the transient dynamics of molecular devices from first principles can be addressed by numerically solving scattering wave function with the detailed algorithm discussed in Ref. 24 or the nonequilibrium Green's function (NEGF) (with numerical details given in Ref. 31) combined with time-dependent density functional theory (TDDFT). ${ }^{32}$ These methods again are very time consuming for the transient current calculation although the scaling has been reduced from $N^{3}$ to $N^{2}\left(\log _{2} N\right)^{2}$, where $N$ is the number of time steps. Therefore, to speed up the calculation, various approximate schemes were proposed to calculate time-dependent transient current of molecular devices such as wide band approximation. ${ }^{28}$ Another approximate scheme based on the exact NEGF solution was also proposed and applied to calculate the transient current of molecular devices which is very efficient and goes beyond the wideband limit. ${ }^{30}$ Despite these efforts, the time-dependent calculation of the transient current for molecular devices is still a challenge on the computational resources. Due to the importance of molecular electronics, it is timely to overcome this problem so that realistic transient dynamics calculations can be performed on molecular devices from first principles. In this paper, we propose a linear scaling $O(N)$ scheme to calculate the timedependent transient current by combining complex absorbing potential (CAP) method with exact solution based on $\mathrm{NEGF}^{25}$ and DFT theory (NEGF-DFT-CAP).

The CAP was initially used to simulate the time-dependent evolution of wave function of finite systems in one and two dimensions. ${ }^{33}$ Recently, CAP was employed to study the transport problem of molecular devices from first principles ${ }^{34-37}$ using a transmission free CAP ${ }^{38}$ By adding an explicitly energy-independent CAP in lead regions, the transport problem in a infinite open system can be reduced to that of a finite simulation region. Moreover, one can obtain an effective wideband-like formula to calculate DC transport quantities such as the transmission coefficient. We note that in general $\mathrm{AC}$ transport properties do not assume the wideband form (effective self-energy does not depend on the energy) in the presence of CAP. Fortunately, for the step-like pulse, we are able to cast the exact NEGF solution for transient current into a wideband form using CAP which enables us to speed up the 
calculation tremendously. In fact, when CAP is implemented into the exact NEGF solution the amount of calculation scales like $c N n^{3}$ where $N$ is the number of time steps, $n$ is the dimension of the Hamiltonian in the whole simulation region including the CAP region, and $c$ is a constant of order of a few hundred. With this order $N$ method at hand, the first-principles transient current calculation of realistic molecular devices is within the reach. We have applied our formalism to molecular devices and carried out the benchmark calculation for transient current on a one-dimensional atomic chain which agrees with the result from exact numerical calculation. Furthermore, we have investigated transient dynamics of a three-dimensional (3D) molecular device and calculated transient current at two different bias voltages. It was found that the transient current involves many time scales showing that the wideband limit is not a good approximation for molecular devices.

The paper is organized as follows. In Sec. II, we will first introduce the formalism of the complex absorbing potential (CAP) and briefly discuss its application in DC transport calculations. Then we will discuss how to apply the CAP to calculate the time-dependent transient current of molecular devices under upward step-like pulse. In Sec. III, benchmark comparisons with the NEGF-DFT method is presented. Then the numerical calculation of the transient current of the $\mathrm{Al}-\mathrm{C}_{3}-\mathrm{Al}$ molecular device is given. Finally, Sec. IV gives discussion and conclusion.

\section{THEORETICAL FORMALISM}

\section{A. Complex absorbing potential}

As shown in Fig. 1, a typical two terminal device consists of a central scattering region connected by two semi-infinite external leads along the transport $z$ direction. The corresponding Hamiltonian of the whole system can be expressed as a tridiagonal block matrix

$$
H=\left[\begin{array}{ccc}
H_{L L} & H_{L C} & 0 \\
H_{C L} & H_{C C} & H_{C R} \\
0 & H_{R C} & H_{R R}
\end{array}\right],
$$

where $H_{\alpha \alpha}, \alpha=L, R$ is the semi-infinite Hamiltonian of the lead $\alpha$. To study transport properties of this open system, one is actually solving the scattering problem with infinite degrees of freedom. In the framework of the NEGF, one calculates various Green's functions of the central region and the effect of leads is taken into account by the self-energy. For instance, the retarded Green's function of the central region in an energy domain is defined as

$$
G_{C C}^{r}(E)=\left(E-H_{C C}-\sum_{\alpha=L, R} \Sigma_{\alpha}^{r}(E)\right)^{-1},
$$

where $\Sigma_{\alpha}^{r}(E)$ is the self-energy of lead $\alpha$

$$
\Sigma_{\alpha}^{r}(E)=H_{C \alpha} g_{\alpha \alpha}^{r}(E) H_{\alpha C},
$$

and $g_{\alpha \alpha}^{r}(E)$ is the retarded Green's function of the corresponding lead $\alpha$

$$
g_{\alpha \alpha}^{r}(E)=\left(E-H_{\alpha \alpha}+i 0^{+}\right)^{-1} .
$$

After obtaining the retarded Green's function, one can calculate various transport quantities, such as the transmission

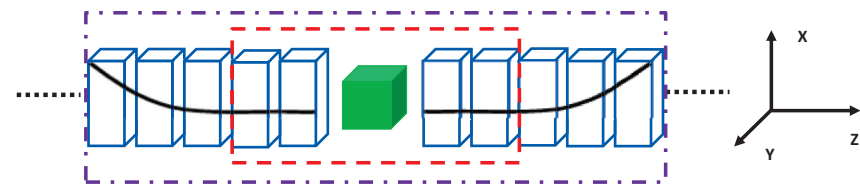

FIG. 1. (Color online) Schematic plot of a two terminal molecular device. The device consists of a central molecular part (green solid cube) and two semi-infinite leads, which will extend to the $\pm \infty$. The black solid lines represent the complex absorbing potential added to both lead regions. The region enclosed by the red dashed line is the central region; the purple dashed-dot line encloses the central region plus complex absorbing potential region in the leads.

coefficient

$$
T(E)=\operatorname{Tr}\left[\Gamma_{L} G_{C C}^{r} \Gamma_{R} G_{C C}^{a}\right] .
$$

Here $\Gamma_{\alpha}(E)=i\left[\Sigma_{\alpha}^{r}(E)-\Sigma_{\alpha}^{a}(E)\right]$ is the linewidth function of the lead $\alpha$. In the numerical calculation, the energy-dependent self-energy can be calculated using the iterative or quadratic eigenvalue approaches. ${ }^{39,40}$ To distinguish it from the CAP method, we will refer to the above method as the exact method.

The idea of the CAP method is to replace the infinite system by a finite system using the complex absorbing potential that absorbs the incident wave function completely. In the application of CAP to the quantum transport problem, CAP is added to a finite lead region (called the CAP region) outside of the central scattering region. Usually, the effectiveness of CAP on absorbing the incident wave depends on the length of the CAP region. The reduction of reflection can be improved by increasing the length of the CAP region in a controlled way. Note that the advantage of the CAP method over the exact method relies on the fact that the CAP does not depends on energy while the self-energy of the exact method does. Using this property, the poles of the Green's function can be obtained easily when the CAP method is used. Therefore the convergence problem of the energy integral in calculating transient current is solved. In the numerical calculation, we adopt an optimized transmission-free CAP given in Ref. 38

$$
W(z)=\frac{\hbar^{2}}{2 m}\left(\frac{2 \pi}{\Delta z}\right)^{2} f(z)
$$

where $f(z)$ is defined as

$$
f(z)=\frac{4}{c^{2}}\left[\left(\frac{\Delta z}{z_{2}-2 z_{1}+z}\right)^{2}+\left(\frac{\Delta z}{z_{2}-z}\right)^{2}-2\right],
$$

and $\Delta z=z_{2}-z_{1}$ is the range of CAP along the transport $z$ direction, $z_{1}$ and $z_{2}$ are the starting and ending points of the CAP region at each lead, respectively. Here $c$ is a constant taken to be 2.62, $m$ is the mass of the electron. As shown in Fig. 1, the CAP region starts from several buffer layers away from the central molecular region. Going deep into the lead, the strength of the absorbing potential increases and $f(z) \rightarrow \infty$ when $z$ approaches the end point $z_{2}$. This truncates the semi-infinite lead into a finite one. Therefore, the numerical simulation region becomes finite, i.e., the region enclosed by the purple dashed-dot line in Fig. 1. In the first-principles calculation, a linear combination of atomic orbitals (LCAO) basis set is usually adopted. Then one has to calculate the matrix element 
of the CAP in orbital space

$$
W_{\mu \nu}=\int \phi_{\mu}^{*}(x, y, z) W(z) \phi_{\nu}(x, y, z) d x d y d z,
$$

where $\phi_{\mu}$ is the atomic orbital.

Within the CAP method (all quantities are labeled with a prime), the retarded Green's function of lead $\alpha$ can be defined as $^{36}$

$$
g_{\alpha \alpha}^{r^{\prime}}(E)=\left(E-H_{\alpha \alpha}+i W_{\alpha}\right)^{-1} .
$$

Since the lead is effectively truncated, $H_{\alpha \alpha}^{\prime}=H_{\alpha \alpha}-i W_{\alpha}$ is a matrix of finite dimension shown in Fig. 1. The retarded Green's function of the whole system including the CAP region can be expressed as

$$
G^{r^{\prime}}=\left(E-H+i \sum_{\alpha} W_{\alpha}^{\prime}\right)^{-1},
$$

with $W_{L}^{\prime}=\left[\begin{array}{ccc}W_{L} & 0 & 0 \\ 0 & 0 & 0 \\ 0 & 0 & 0\end{array}\right]$ and $W_{R}^{\prime}=\left[\begin{array}{ccc}0 & 0 & 0 \\ 0 & 0 & 0 \\ 0 & 0 & W_{R}\end{array}\right]$.

Although the lead region (including the CAP region) is finite, we can still use the concept of self-energy and obtain an effective retarded Green's function of the central region within the CAP method

$$
G_{C C}^{r^{\prime}}=\left(E-H_{C C}-\sum_{\alpha} \Sigma_{\alpha}^{r^{\prime}}\right)^{-1},
$$

where the self-energy $\Sigma_{\alpha}^{r^{\prime}}$ is given by ${ }^{41}$

$$
\Sigma_{\alpha}^{r^{\prime}}(E)=H_{C \alpha} g_{\alpha \alpha}^{r^{\prime}}(E) H_{\alpha C} .
$$

It is easy to show that the linewidth function is written as ${ }^{42}$

$$
\Gamma_{\alpha}^{\prime}=2 H_{C \alpha} g_{\alpha \alpha}^{r^{\prime}} W_{\alpha} g_{\alpha \alpha}^{a^{\prime}} H_{\alpha C}=2 H_{C \alpha} g_{\alpha \alpha}^{a^{\prime}} W_{\alpha} g_{\alpha \alpha}^{r^{\prime}} H_{\alpha C} .
$$

Since the self-energy calculated by the CAP method is the same as that obtained from the traditional method, ${ }^{36}$ various Green's functions in the central (physically relevant) region shown in Fig. 1 should also be the same as that given in Eq. (2). At this level, the self-energy of the Green's function of the central scattering region $G_{C C}^{r^{\prime}}$ depends on energy. In the following, we give a simple derivation to show that the transmission coefficient can be put into an effective wide band limit (WBL) form in the CAP method. Starting from the traditional definition of the transmission coefficient of Eq. (12) together with Eq. (13)

$$
\begin{aligned}
T(E)= & \operatorname{Tr}\left[\Gamma_{L}^{\prime} G_{C C}^{r^{\prime}} \Gamma_{R}^{\prime} G_{C C}^{a^{\prime}}\right] \\
= & 4 \operatorname{Tr}\left[H_{C L} g_{L L}^{a^{\prime}} W_{L} g_{L L}^{r^{\prime}} H_{L C} G_{C C}^{r^{\prime}} H_{C R} g_{R R}^{r^{\prime}}\right. \\
& \left.\times W_{R} g_{R R}^{a^{\prime}} H_{R C} G_{C C}^{a^{\prime}}\right] \\
= & 4 \operatorname{Tr}\left[W_{L} G_{L R}^{r^{\prime}} W_{R} G_{R L}^{a^{\prime}}\right]=4 \operatorname{Tr}\left[W_{L}^{\prime} G^{r^{\prime}} W_{R}^{\prime} G^{a^{\prime}}\right],
\end{aligned}
$$

where we have defined the following Green's function of the whole system including the CAP region (see Appendix A for the derivation)

$$
G_{L R}^{r^{\prime}}=g_{L L}^{r^{\prime}} H_{L C} G_{C C}^{r^{\prime}} H_{C R} g_{R R}^{r^{\prime}}
$$

To calculate the transmission coefficient of Eq. (14), one only needs to know $G^{r^{\prime}}$, which is defined in the whole system including the CAP region with $W_{\alpha}^{\prime}$ an effective energyindependent self-energy. Note that this effective WBL form is only valid in the DC case. In the case of AC transport, one may not have a similar WBL form and one has to deal with it case by case.

In terms of the lesser Green's function, one can calculate the charge density in the central region. We also start from the traditional definition of the lesser Green's function

$$
\begin{aligned}
G_{C C}^{<}(E) & =i \sum_{\alpha} f_{\alpha} G_{C C}^{r^{\prime}} \Gamma_{\alpha}^{\prime} G_{C C}^{a^{\prime}} \\
& =2 i \sum_{\alpha} f_{\alpha} G_{C \alpha}^{r^{\prime}} W_{\alpha} G_{\alpha C}^{a^{\prime}} \\
& =2 i \sum_{\alpha} f_{\alpha}\left[G^{r^{\prime}} W_{\alpha}^{\prime} G^{a^{\prime}}\right]_{C C},
\end{aligned}
$$

where we have used Eq. (13) and $G_{C \alpha}^{r^{\prime}}=G_{C C}^{r^{\prime}} H_{C \alpha} g_{\alpha \alpha}^{r^{\prime}}$ [see Eq. (3.5.13) in Ref. 41]; $f_{\alpha}$ is the Fermi distribution function of lead $\alpha$.

\section{B. Time-dependent transient current with upward step-like pulse}

The exact solution of time-dependent current for the steplike pulse based on NEGF has been given by Maciejko et al. ${ }^{25}$ This formalism can be combined with DFT to calculate the transient current in molecular devices. ${ }^{30,43}$ In the following, we will combine the exact solution with DFT and CAP to obtain an order $O(N)$ scheme (NEGF-DFT-CAP) for calculating the time-dependent current under the upward step-like pulse. Downward step-like and square-like pulses can also be treated in a similar fashion.

To begin with, we will derive an equivalent time-dependent current formula. Starting from the equation of motion for the lesser Green's function, ${ }^{44}$ we have

$$
\begin{aligned}
i \frac{\partial}{\partial t} G_{C C}^{<}\left(t, t^{\prime}\right)= & H_{C C}(t) G_{C C}^{<}\left(t, t^{\prime}\right)+\int_{0}^{t}\left[\Sigma^{<}\left(t, t_{1}\right) G_{C C}^{a}\left(t_{1}, t^{\prime}\right)\right. \\
& \left.+\Sigma^{r}\left(t, t_{1}\right) G^{<}\left(t_{1}, t^{\prime}\right)\right] d t_{1},
\end{aligned}
$$

and

$$
\begin{aligned}
-i \frac{\partial}{\partial t^{\prime}} G_{C C}^{<}\left(t, t^{\prime}\right)= & G_{C C}^{<}\left(t, t^{\prime}\right) H_{C C}\left(t^{\prime}\right)+\int_{0}^{t}\left[G_{C C}^{r}\left(t, t_{1}\right) \Sigma^{<}\left(t_{1}, t^{\prime}\right)\right. \\
& \left.+G^{<}\left(t, t_{1}\right) \Sigma^{a}\left(t_{1}, t^{\prime}\right)\right] d t_{1} .
\end{aligned}
$$

Then subtracting Eq. (18) by Eq. (17) and setting $t^{\prime}=t$, we can arrive at

$$
I_{o p}(t)=H_{C C}(t) G_{C C}^{<}(t, t)-G_{C C}^{<}(t, t) H_{C C}(t)-i \frac{\partial}{\partial t} G_{C C}^{<}(t, t),
$$

where we have defined

$$
\begin{aligned}
I_{o p}(t) \equiv & \int_{0}^{t}\left[G_{C C}^{r}\left(t, t_{1}\right) \Sigma^{<}\left(t_{1}, t\right)+G^{<}\left(t, t_{1}\right) \Sigma^{a}\left(t_{1}, t\right)\right. \\
& \left.-\Sigma^{<}\left(t, t_{1}\right) G_{C C}^{a}\left(t_{1}, t\right)-\Sigma^{r}\left(t, t_{1}\right) G^{<}\left(t_{1}, t\right)\right] d t_{1},
\end{aligned}
$$

which is a matrix. Note that the terminal current $I_{\alpha}(t)$ (Ref. 45) can be obtained from $I_{o p}(t)$. To do that, two auxiliary projection 
matrices are introduced

$$
\bar{\Gamma}_{L}=\left[\begin{array}{ccc}
1_{L} & 0 & 0 \\
0 & 0 & 0 \\
0 & 0 & 0
\end{array}\right], \quad \bar{\Gamma}_{R}=\left[\begin{array}{ccc}
0 & 0 & 0 \\
0 & 0 & 0 \\
0 & 0 & 1_{R}
\end{array}\right],
$$

where $\alpha$ denotes the outermost unit cell layer in buffer layers of the central region and $1_{L / R}$ is the unit matrix with a dimension equal to the size of unit cell of left and right leads, respectively, so that $\Sigma_{L}^{r}=\bar{\Gamma}_{L} \Sigma^{r} \bar{\Gamma}_{L}$. Finally we have

$$
I_{\alpha}(t)=\operatorname{Tr}\left[\bar{\Gamma}_{\alpha} I_{o p}(t) \bar{\Gamma}_{\alpha}\right] .
$$

From Eq. (19), we can use the following formula to calculate the time-dependent terminal current $I_{\alpha}(t)$,

$$
\begin{aligned}
I_{\alpha}(t)= & 2 \operatorname{Re} \operatorname{Tr}\left[\bar{\Gamma}_{\alpha} H_{C C}(t) G_{C C}^{<}(t, t) \bar{\Gamma}_{\alpha}\right] \\
& -i \operatorname{Tr}\left[\bar{\Gamma}_{\alpha} \partial_{t} G_{C C}^{<}(t, t) \bar{\Gamma}_{\alpha}\right],
\end{aligned}
$$

where $G_{C C}^{<}(t, t)$ is the time-dependent lesser Green's function of the central region with equal time. To calculate $I_{\alpha}(t)$, one has to know the time-dependent Hamiltonian $H(t)$ and calculate the time-dependent lesser Green's function $G_{C C}^{<}(t, t)$. Since the external bias is the upward step-like pulse in our problem, then the time-dependent Hamiltonian can be obtained as follows. When time $t<0, H(t<0)=H_{e q}$ is an equilibrium Hamiltonian without bias and $H(t \geqslant 0)=\theta(t) H_{n e q}$ that $H_{n e q}$ is the self consistent nonequilibrium Hamiltonian under DC bias. As for the time-dependent lesser Green's function within the CAP method, it can be written as

$$
G_{C C}^{<}(t, t)=2 i \sum_{\alpha} \int \frac{d \omega}{2 \pi} f(\omega)\left[A_{\alpha}^{\prime}(\omega, t) W_{\alpha}^{\prime} A_{\alpha}^{\prime \dagger}(\omega, t)\right]_{C C},
$$

where we have used the spectral function $A_{\alpha}^{\prime}(\omega, t)$ (Ref. 45)

$$
A_{\alpha}^{\prime}(\epsilon, t) \equiv \int_{-\infty}^{t} d t^{\prime} e^{i \epsilon\left(t-t^{\prime}\right)} e^{i \int_{t^{\prime}}^{t} d t_{1} \Delta_{\alpha}\left(t_{1}\right)} G^{r^{\prime}}\left(t, t^{\prime}\right),
$$

where $\Delta_{\alpha}(t)$ is the time-dependent external bias. Note that $A_{\alpha}^{\prime}(\epsilon, t)$ has the same dimension as $G^{r^{\prime}}$ that is defined in the CAP method. Then the key issue here is how to calculate quantity $A_{\alpha}^{\prime}(\epsilon, t)$ efficiently. From the analytic expression of $A_{\alpha}(\epsilon, t)$ given in Ref. 25, we can derive the spectral function in the CAP form (see Appendix B for the derivation),

$$
\begin{aligned}
A_{\alpha}^{\prime}(\epsilon, t)= & \bar{G}^{r^{\prime}}\left(\epsilon+\Delta_{\alpha}\right)-\int \frac{d \omega}{2 \pi i} \frac{e^{-i(\omega-\epsilon) t} \bar{G}^{r^{\prime}}\left(\omega+\Delta_{\alpha}\right)}{\omega-\epsilon+\Delta_{\alpha}-i 0^{+}} \\
& \times\left[\frac{\Delta_{\alpha}}{\omega-\epsilon-i 0^{+}}+\Delta \tilde{G}^{r^{\prime}}(\epsilon)\right],
\end{aligned}
$$

where $\Delta=H_{n e q}-H_{e q}$ is the internal potential change due to the external bias. The quilibrium and nonequilibrium retarded Green's functions are defined as

$$
\begin{aligned}
& \tilde{G}^{r^{\prime}}(\epsilon)=\left[\epsilon I-H_{e q}+i \sum_{\alpha} W_{\alpha}^{\prime}\right]^{-1}, \\
& \bar{G}^{r^{\prime}}(\epsilon)=\left[\epsilon I-H_{n e q}+i \sum_{\alpha} W_{\alpha}^{\prime}\right]^{-1} .
\end{aligned}
$$

Since $W_{\alpha}^{\prime}$ is energy independent, we can use following eigenequations to construct the retarded Green's functions,

$$
\begin{gathered}
{\left[H_{n e q}-i \sum_{\alpha} W_{\alpha}^{\prime}\right]\left|\psi_{n}\right\rangle=\epsilon_{n}\left|\psi_{n}\right\rangle,} \\
{\left[H_{n e q}+i \sum_{\alpha}\left(W_{\alpha}^{\prime}\right)^{\dagger}\right]\left|\phi_{n}\right\rangle=\epsilon_{n}^{*}\left|\phi_{n}\right\rangle,}
\end{gathered}
$$

and

$$
\begin{gathered}
{\left[H_{e q}-i \sum_{\alpha} W_{\alpha}^{\prime}\right]\left|\psi_{n}^{0}\right\rangle=\epsilon_{n}^{0}\left|\psi_{n}^{0}\right\rangle,} \\
{\left[H_{e q}+i \sum_{\alpha}\left(W_{\alpha}^{\prime}\right)^{\dagger}\right]\left|\phi_{n}^{0}\right\rangle=\epsilon_{n}^{0 *}\left|\phi_{n}^{0}\right\rangle .}
\end{gathered}
$$

Then retarded Green's functions can be constructed from their eigenfunctions

$$
\begin{aligned}
\tilde{G}^{r^{\prime}}(\epsilon) & =\sum_{n} \frac{\left|\psi_{n}^{0}\right\rangle\left\langle\phi_{n}^{0}\right|}{\left(\epsilon-\epsilon_{n}^{0}+i 0^{+}\right)}, \\
\bar{G}^{r^{\prime}}(\epsilon) & =\sum_{n} \frac{\left|\psi_{n}\right\rangle\left\langle\phi_{n}\right|}{\left(\epsilon-\epsilon_{n}+i 0^{+}\right)} .
\end{aligned}
$$

Due to the presence of the time-dependent factor $e^{-i(\omega-\epsilon) t}$ in $A_{\alpha}^{\prime}$, the integration in $A_{\alpha}^{\prime}(\epsilon, t)$ can be done analytically by enclosing a contour in the lower half of the complex plane

$$
\begin{aligned}
& A_{\alpha}^{\prime}(\epsilon, t) \\
& =\sum_{n} \frac{\left|\psi_{n}\right\rangle\left\langle\phi_{n}\right|}{\left(\epsilon+\Delta_{\alpha}-\epsilon_{n}+i 0^{+}\right)}+\sum_{n} \frac{e^{i\left(\epsilon+\Delta_{\alpha}-\epsilon_{n}\right) t}\left|\psi_{n}\right\rangle\left\langle\phi_{n}\right|}{\epsilon-\epsilon_{n}+i 0^{+}} \\
& \quad \times\left[\frac{\Delta_{\alpha}}{\epsilon+\Delta_{\alpha}-\epsilon_{n}+i 0^{+}}-\Delta \sum_{m} \frac{\left.\mid \psi_{m}^{0}\right)\left\langle\phi_{m}^{0}\right|}{\left(\epsilon-\epsilon_{m}^{0}+i 0^{+}\right)}\right] .
\end{aligned}
$$

It is easy to check that in the initial state and asymptotic long time limit $(t \rightarrow \infty) A_{\alpha}^{\prime}(t)$ is equal to $\tilde{G}^{r^{\prime}}(\epsilon)$ and $\bar{G}^{r^{\prime}}(\epsilon)$, respectively. After obtaining the $A_{\alpha}^{\prime}(t)$, one can calculate the lesser Green's function using Eq. (24) and hence the timedependent current from Eq. (23). Since $A_{\alpha}^{\prime}(t)$ is expressed as a summation form at any given time $t$, one only needs to integrate the energy $\omega$ in Eq. (24) to obtain the time-dependent lesser Green's function and hence the transient current $I_{\alpha}(t)$. We can estimate the number of operations in calculating the time-dependent current $I_{\alpha}(t)$. For a given time, the calculation only involves matrix multiplication as well as the integral over $\omega$ which again can be done using the theorem of residue. Hence the total number of operations is roughly $c N^{3}$ which is an order $N$ algorithm, where $c$ is of order 200 due to the contour integral on the complex plane, $n$ is the dimension of the Green's function of the whole system including the CAP region, and $N$ is the number of time steps. To make the algorithm more efficient, we need to keep $n$ as small as possible. Clearly, for molecular devices with large degrees of freedom, adding a CAP region will not increase $n$ very much. Hence the strength of this algorithm is for large-scale calculations. In addition, the CAP region can also be optimized. As demonstrated in Ref. 36, the number of CAP unit cells can be reduced to six for each lead as compared with the 30 unit cells used in the calculation below. 


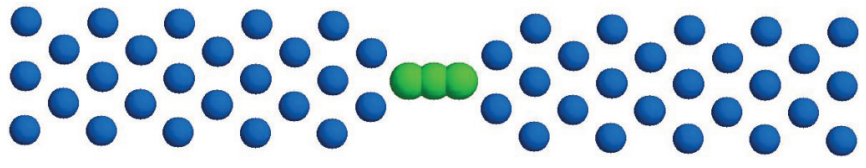

FIG. 2. (Color online) Schematic diagram of a molecular device $\mathrm{Al}-\mathrm{C}_{3}-\mathrm{Al}$. The device consists of a three carbon atoms chain coupled to the perfect aluminium atomic electrodes which extends to the reservoirs at $\pm \infty$, where the current is collected.

The major steps for the numerical calculation can be summarized as follows. We first prepare the initial equilibrium and final nonequilibrium self-consistent Hamiltonian from DC calculations. Then we construct the CAP matrix $W_{\alpha}$ with respective to the lead. Once the CAP is constructed, one has to compare the transmission coefficients with that obtained by the exact method to get an idea of how long the CAP region should be. With the good agreement on the transmission coefficient, we can move on to calculate the time-dependent current using $A_{\alpha}^{\prime}(t)$.

It is worth mentioning that, in the above discussion, the orthogonal basis set is implicitly assumed to expand the Hamiltonian. So one has to orthogonalize the basis set if a nonorthogonal basis such as an atomic orbital basis set (LCAO) is used. ${ }^{43}$

\section{NUMERICAL RESULTS}

In this section, the implementation of our formalism and numerical results of the transient current for the $\mathrm{Al}-\mathrm{C}-\mathrm{Al}$ molecular device will be presented. The structure of the $\mathrm{Al}-\mathrm{C}_{3}-\mathrm{Al}$ molecular device is shown in Fig. 2. There are 75 atoms in the central scattering region and the distance between the $\mathrm{Al}$ atom and the nearest carbon atom is equal to 3.78 a.u. As for the electrodes, there are nine aluminum atoms in a unit cell with a finite cross section along (100) direction in the semi-infinite aluminum lead. Our numerical analysis is based on the state-of-the-art first-principles quantum transport package MATDCAL. ${ }^{46,47}$ Specifically, a linear combination of atomic orbitals (LCAO) is employed to solve Kohn-Sham equations. The exchange-correlation is treated at the local density approximation level and the nonlocal norm-conserving pseudopotential ${ }^{48}$ is used to define the atomic core. The density matrix is constructed in orbital space and the effective potential is obtained in real space by solving the Poisson equation. The self-consistent NEGF-DFT iteration in MATDCAL package was carried out until the numerical tolerance is less than $10^{-4} \mathrm{eV}$. The initial equilibrium and final nonequilibrium Hamiltonian were prepared using the MATDCAL package.

In the following, the case of an upward step-like pulse $\left[V_{L}(t)=-V_{R}(t)=\theta(t) V\right]$ applied on both leads will be considered. To satisfy the current conservation condition, we will plot the time-dependent current in terms of $I(t)=$ $\left[I_{L}(t)-I_{R}(t)\right] / 2$ (Ref. 43).

To test the accuracy of our present scheme, we have calculated the transient current using two different approaches. One is based on the NEGF-DFT method proposed in Ref. 31 which is an order $N^{2}\left(\log _{2} N\right)^{2}$ algorithm and other one is our proposed formalism in this paper termed as NEGF-DFT-CAP. Here we take a one-dimensional $\mathrm{Al}-\mathrm{C}_{1}-\mathrm{Al}$ atomic chain

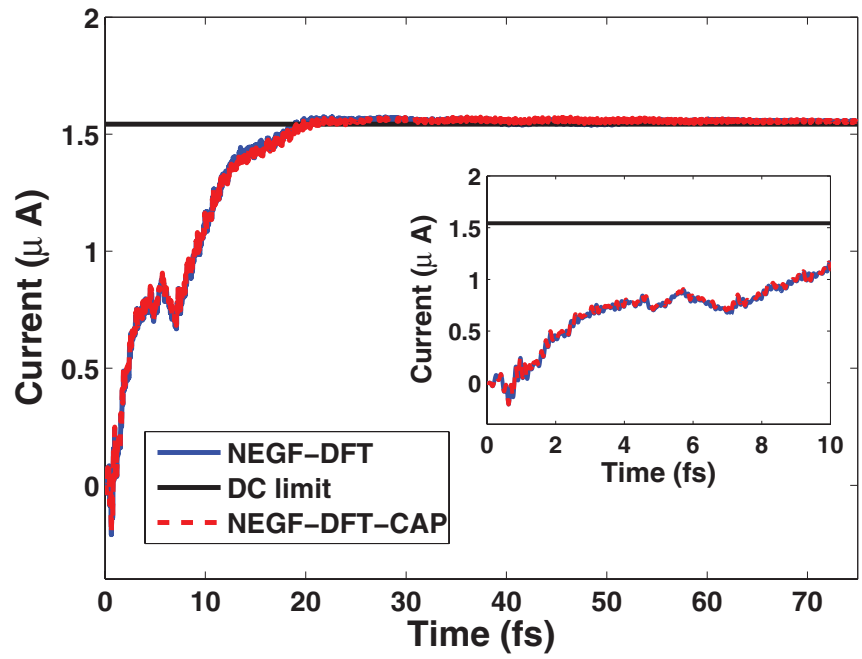

FIG. 3. (Color online) The time-dependent transient current $I(t)$ versus time with $V=0.0005$ a.u. for one-dimensional atomic $\mathrm{Al}-$ $\mathrm{C}_{1}-\mathrm{Al}$ chain. Blue solid line and red dashed line are time-transientdependent current calculated by using NEGF-DFT method and NEGF-DFT-CAP method; black solid line is the DC current at the steady state limit.

(where both leads are a one-dimensional $\mathrm{Al}$ chain) as a toy molecular device and apply a step-like pulse to test the numerical implementation of our formalism. As shown in Fig. 3, the transient current calculated from two different methods agree well with each other. In addition, the transient current approaches to the DC steady state value obtained by using the Landauer-Büttiker formula in the long time limit. The inset of Fig. 3 shows the early time behavior of the transient current.

Now let us study a more realistic model $\mathrm{Al}-\mathrm{C}_{3}-\mathrm{Al}$ system. First of all, we have to compare the transmission coefficient by using the CAP method and exact method to make sure that the CAP potential is added correctly. As you can see in Fig. 4, the CAP result agrees well with that calculated by the exact method.

After examining the accuracy of the transmission coefficient of the CAP method, we are ready to study the transient

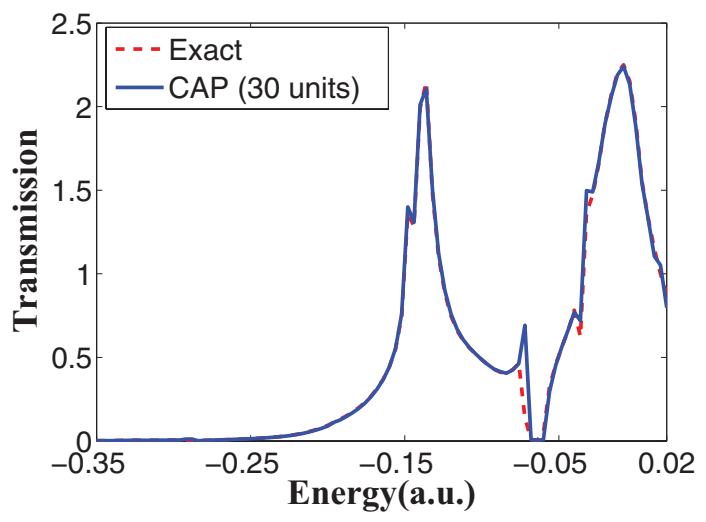

FIG. 4. (Color online) The comparison of the transmission coefficient of a carbon chain sandwiched between $\mathrm{Al}(100)$ leads. The numerical results calculated by using CAP method with 30 unit cells in the lead region (blue solid line) is compared with the exact method (red dashed line). 


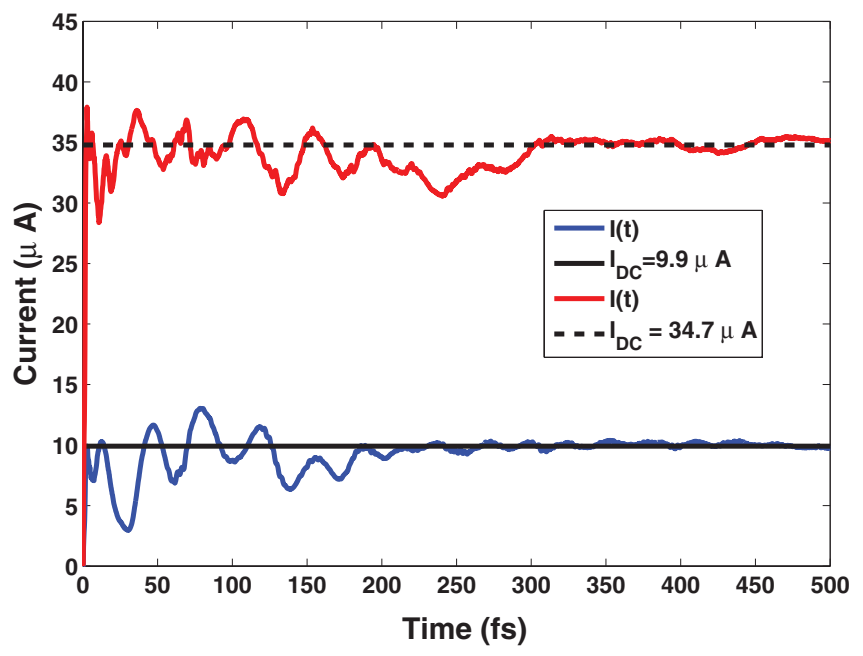

FIG. 5. (Color online) The time-dependent transient current $I(t)$ versus time with different bias voltages for a $\mathrm{Al}-\mathrm{C}_{3}-\mathrm{Al}$ molecular device. The blue and black solid lines correspond to the timedependent transient current and DC current at a steady state for $V=0.0025$ a.u., respectively. The red solid and black dashed lines are the time-dependent transient current and DC current at steady state for $V=0.01$ a.u., respectively.

current of the $\mathrm{Al}-\mathrm{C}_{3}-\mathrm{Al}$ molecular junction. We calculate the transient current under two different bias voltages. The numerical results are plotted in Fig. 5. We have serval observations: (1) the switch-on time is roughly $2 \mathrm{fs}$; (2) the relaxation time is roughly 210 fs for $V=0.0025$ a.u. and 320 fs for $V=0.01$ a.u.; (3) the transient current is on the same order of magnitude as that of the DC steady state limit. In the early time, there are some irregular oscillations in the transient current. At the long time limit, the transient current approaches to the correct DC limit. Moreover, more oscillations occur with the increase of bias voltage. The nature of the oscillation can be attributed to the resonant states of the system. ${ }^{30}$

\section{SUMMARY}

To summarize, we have proposed an order $N$ first-principle formalism to study the dynamical response of molecular devices due to the time-dependent step-like external bias. Our formalism is based on NEGF combined with DFT as well the CAP method. The use of the CAP allows us to calculate the transient current efficiently compared to previous NEGF-DFT schemes. Detailed computational procedures for firstprinciples transient current calculation were discussed which are very easy to implement. As an illustration, we have calculated the transient current of $\mathrm{Al}-\mathrm{C}_{3}-\mathrm{Al}$ molecular devices from first principles. We wish to emphasize that one has to use an orthogonal basis set to make the implementation simple. In addition, our NEGF-DFT-CAP formalism is aimed to study the transient current under step-like pulse. In addition, we have assumed that the Coulomb interaction changes instantly with the external bias, i.e., the time-dependent Hamiltonian $H(t \geqslant$ $0)=\theta(t) H_{\text {neq }}$, where $H_{\text {neq }}$ is calculated from the NEGF-DFT formalism. We hope to go beyond this approximation in future works.

\section{ACKNOWLEDGMENTS}

We gratefully acknowledge the support from Research Grant Council (HKU 705611P) and University Grant Council (Contract No. AoE/P-04/08) of the Government of HKSAR. This research was conducted using the HKU Computer Centre research computing facilities that are supported in part by the Hong Kong UGC Special Equipment Grant (SEG HKU09).

\section{APPENDIX A: DERIVATIONS FOR $G_{L R}^{r^{\prime}}$}

According to the definition of the retarded Green's function,

$$
\begin{aligned}
& {\left[\begin{array}{ccc}
\left(g_{L L}^{r^{\prime}}\right)^{-1} & -H_{L C} & 0 \\
-H_{C L} & E-H_{C C} & -H_{C R} \\
0 & -H_{R C} & \left(g_{R R}^{r^{\prime}}\right)^{-1}
\end{array}\right]\left[\begin{array}{ccc}
G_{L L}^{r^{\prime}} & G_{L C}^{r^{\prime}} & G_{L R}^{r^{\prime}} \\
G_{C L}^{r^{\prime}} & G_{C C}^{r^{\prime}} & G_{C R}^{r^{\prime}} \\
G_{R L}^{r^{\prime}} & G_{R C}^{r^{\prime}} & G_{R R}^{r^{\prime}}
\end{array}\right]} \\
& =\left[\begin{array}{ccc}
1_{L} & 0 & 0 \\
0 & 1_{C} & 0 \\
0 & 0 & 1_{R}
\end{array}\right],
\end{aligned}
$$

we have

$$
G_{L R}^{r^{\prime}}=g_{L L}^{r^{\prime}} H_{L C} G_{C R}^{r^{\prime}} .
$$

To find $G_{C R}^{r^{\prime}}$ we note that the advanced Green's function can be obtained by changing superscript $r$ into $a$ in Eq. (A1). We have

$$
G_{R C}^{a^{\prime}}=g_{R R}^{a^{\prime}} H_{R C} G_{C C}^{a^{\prime}}, \quad G_{C R}^{r^{\prime}}=G_{C C}^{r^{\prime}} H_{C R} g_{R R}^{r^{\prime}},
$$

where we have used the fact that $G_{C R}^{r^{\prime}}=\left(G_{R C}^{a^{\prime}}\right)^{\dagger}$. Finally we combine Eqs. (A1) and (A3) to arrive at

$$
G_{L R}^{r^{\prime}}=g_{L L}^{r^{\prime}} H_{L C} G_{C C}^{r^{\prime}} H_{C R} g_{R R}^{r^{\prime}}
$$

\section{APPENDIX B: DERIVATIONS FOR $A_{\alpha_{C C}}^{\prime}$}

In this Appendix, we will derive the expression of $A_{\alpha_{C C}}^{\prime}$ in the central region within the CAP method. Starting from Eq. (26), we have

$$
\begin{aligned}
A_{\alpha_{C C}}^{\prime}(\epsilon, t)= & \bar{G}_{C C}^{r^{\prime}}\left(\epsilon+\Delta_{\alpha}\right)-\int \frac{d \omega}{2 \pi i} \frac{e^{-i(\omega-\epsilon) t}}{\omega-\epsilon+\Delta_{\alpha}-i 0^{+}} \\
& \times\left[\bar{G}_{C C}^{r^{\prime}}\left(\omega+\Delta_{\alpha}\right) \frac{\Delta_{\alpha}}{\omega-\epsilon-i 0^{+}}+B_{0}\right],
\end{aligned}
$$

with

$$
B_{0} \equiv \sum_{\beta=L, C, R} \bar{G}_{C \beta}^{r^{\prime}}\left(\omega+\Delta_{\alpha}\right) \Delta_{\beta \beta} \tilde{G}_{\beta C}^{r^{\prime}}(\epsilon),
$$

where $\Delta_{C C}$ is the internal potential change in the central region due to the external bias in the leads and $\Delta_{\beta \beta}=\Delta_{\beta} 1_{\beta}$ with $\beta=L, R$ is the bias applied in the lead $\beta$. Furthermore, $B_{0}$ can be separated into two parts,

$$
\begin{aligned}
B_{0} & =\bar{G}_{C C}^{r^{\prime}} \Delta_{C C} \tilde{G}_{C C}^{r^{\prime}}+\sum_{\beta=L, R} \Delta_{\beta} \bar{G}_{C \beta}^{r^{\prime}} \tilde{G}_{\beta C}^{r^{\prime}} \\
& =\bar{G}_{C C}^{r^{\prime}} \Delta_{C C} \tilde{G}_{C C}^{r^{\prime}}+\sum_{\beta=L, R} \Delta_{\beta} \bar{G}_{C C}^{r^{\prime}} H_{C \beta} \bar{g}_{\beta \beta}^{r^{\prime}} \tilde{g}_{\beta \beta}^{r^{\prime}} H_{\beta C} \tilde{G}_{C C}^{r^{\prime}},
\end{aligned}
$$

where we have used $\bar{G}_{C \beta}^{r^{\prime}}=\bar{G}_{C C}^{r^{\prime}} H_{C \beta} \bar{g}_{\beta \beta}^{r^{\prime}}$ and $\tilde{G}_{\beta C}^{r^{\prime}}=$ $\tilde{g}_{\beta \beta}^{r^{\prime}} H_{\beta C} \tilde{G}_{C C}^{r^{\prime}}$. According to the retarded Green's function of 
the lead $\alpha$ in Eq. (9), we have

$$
\begin{aligned}
\bar{g}_{\beta \beta}^{r^{\prime}} \tilde{g}_{\beta \beta}^{r^{\prime}}= & \frac{1}{\omega+\Delta_{\alpha}-\Delta_{\beta}-H_{\beta \beta}^{\prime}} \frac{1}{\epsilon-H_{\beta \beta}^{\prime}} \\
= & \frac{1}{\epsilon-\omega-\Delta_{\alpha}+\Delta_{\beta}} \\
& \times\left[\frac{1}{\omega+\Delta_{\alpha}-\Delta_{\beta}-H_{\beta \beta}^{\prime}}-\frac{1}{\epsilon-H_{\beta \beta}^{\prime}}\right] .
\end{aligned}
$$

Therefore, $B_{0}$ becomes

$$
B_{0}=\bar{G}_{C C}^{r^{\prime}} \Delta_{C C} \tilde{G}_{C C}^{r^{\prime}}-\sum_{\beta=L, R} \Delta_{\beta} \bar{G}_{C C}^{r^{\prime}} \tilde{\Upsilon}_{\alpha \beta}^{R}(\epsilon, \omega) \tilde{G}_{C C}^{r^{\prime}},
$$

where we have defined

$$
\tilde{\Upsilon}_{\alpha \beta}^{R}(\epsilon, \omega) \equiv \frac{\tilde{\Sigma}_{\beta}^{R^{\prime}}(\epsilon)-\tilde{\Sigma}_{\beta}^{R^{\prime}}\left(\omega+\Delta_{\alpha}-\Delta_{\beta}\right)}{\epsilon-\omega-\Delta_{\alpha}+\Delta_{\beta}} .
$$

Finally, plugging Eq. (B4) into Eq. (B1), one can easily find that the final expression for $A_{\alpha_{C C}}^{\prime}$ is the same as the expression for $A_{\alpha_{C C}}$ given in Ref. 25. *jianwang@hku.hk

${ }^{1}$ A. Aviram and M. Ratner, Chem. Phys. Lett. 29, 277 (1974).

${ }^{2}$ J. Chen, M. A. Reed, A. M. Rawlett, and J. M. Tour, Science 286, 1550 (1999).

${ }^{3}$ C. P. Collier, E. W. Wong, M. Belohradský, F. M. Raymo, J. F. Stoddart, P. J. Kuekes, R. S. Williams, and J. R. Heath, Science 285, 391 (1999).

${ }^{4}$ C. Joachim, J. K. Gimzewski, and A. Aviram, Nature (London) 408, 541 (2000).

${ }^{5}$ J. R. Heath and M. A. Ratner, Phys. Today 56, 43 (2003).

${ }^{6}$ J. R. Petta, S. K. Slater, and D. C. Ralph, Phys. Rev. Lett. 93, 136601 (2004).

${ }^{7}$ N. J. Tao, Nature Nanotechnology 1, 173 (2006).

${ }^{8}$ M. Di Ventra, S. T. Pantelides, and N. D. Lang, Phys. Rev. Lett. 84, 979 (2000).

${ }^{9}$ J. Taylor, H. Guo, and J. Wang, Phys. Rev. B 63, 245407 (2001); 63, 121104 (2001).

${ }^{10}$ M. Brandbyge, J. L. Mozos, P. Ordejon, J. Taylor, and K. Stokbro, Phys. Rev. B 65, 165401 (2002).

${ }^{11}$ Y. Xue, S. Datta, and M. A. Ratner, Chem. Phys. 281, 151 (2002).

${ }^{12}$ C. C. Kaun, B. Larade, and H. Guo, Phys. Rev. B 67, 121411(R) (2003).

${ }^{13}$ C. C. Kaun and H. Guo, Nano Lett. 3, 1521 (2003).

${ }^{14}$ T. Frederiksen, M. Brandbyge, N. Lorente, and A.-P. Jauho, Phys. Rev. Lett. 93, 256601 (2004).

${ }^{15}$ T. Lee, W. Wang, J. F. Klemic, J. J. Zhang, J. Su, and M. A. Reed, J. Phys. Chem. B 108, 8742 (2004).

${ }^{16}$ C. Toher, A. Filippetti, S. Sanvito, and Kieron Burke, Phys. Rev. Lett. 95, 146402 (2005).

${ }^{17}$ M. Koentopp, K. Burke, and F. Evers, Phys. Rev. B 73, 121403(R) (2006).

${ }^{18}$ G. Stefanucci and S. Kurth, Phys. Rev. Lett. 107, 216401 (2011).

${ }^{19}$ J. P. Bergfield, Z. F. Liu, K. Burke, and C. A. Stafford, Phys. Rev. Lett. 108, 066801 (2012).

${ }^{20}$ P. Tröster, P. Schmitteckert, and F. Evers, Phys. Rev. B 85, 115409 (2012).

${ }^{21}$ N. S. Wingreen, A.-P. Jauho, and Y. Meir, Phys. Rev. B 48, 8487 (1993).

${ }^{22}$ M. Di Ventra and T. N. Todorov, J. Phys.: Condens. Matter 16, 8025 (2004).
${ }^{23}$ G. Stefanucci and C.-O. Almbladh, Phys. Rev. B 69, 195318 (2004)

${ }^{24}$ S. Kurth, G. Stefanucci, C.-O. Almbladh, A. Rubio, and E. K. U. Gross, Phys. Rev. B 72, 035308 (2005).

${ }^{25}$ J. Maciejko, J. Wang, and H. Guo, Phys. Rev. B 74, 085324 (2006).

${ }^{26}$ C.-L. Cheng, J. S. Evans, and T. V. Voorish, Phys. Rev. B 74, 155112 (2006).

${ }^{27}$ G. Stefanucci, S. Kurth, E. K. U. Gross, and A. Rubio, Theor. Comput. Chem. 17, 247 (2007).

${ }^{28}$ X. Zheng, F. Wang, C. Y. Yam, Y. Mo, and G. H. Chen, Phys. Rev. B 75, 195127 (2007).

${ }^{29}$ V. Moldoveanu, V. Gudmundsson, and A. Manolescu, Phys. Rev. B 76, 085330 (2007).

${ }^{30}$ B. Wang, Y. X. Xing, L. Zhang, and J. Wang, Phys. Rev. B 81, 121103(R) (2010).

${ }^{31}$ L. Zhang, Y. X. Xing, and J. Wang, Phys. Rev. B 86, 155438 (2012).

${ }^{32}$ E. Runge and E. K. U. Gross, Phys. Rev. Lett. 52, 997 (1984).

${ }^{33}$ R. Kosloff and D. Kosloff, J. Comput. Phys. 63, 363 (1986).

${ }^{34}$ T. M. Henderson et al., J. Chem. Phys. 125, 244104 (2006).

${ }^{35}$ X.-G. Zhang, K. Varga, and S. T. Pantelides, Phys. Rev. B 76, 035108 (2007)

${ }^{36}$ J. A. Driscoll and K. Varga, Phys. Rev. B 78, 245118 (2008).

${ }^{37}$ K. Varga, Phys. Rev. B 83, 195130 (2011).

${ }^{38}$ T. Gonzalez-Lezena, E. J. Rackham, and D. E. Manolopoulos, J. Chem. Phys. 120, 2247 (2004).

${ }^{39}$ Lopez-Sancho et al., J. Phys. F 14, 1205 (1984); 15, 851 (1985).

${ }^{40}$ S. Sanvito, C. J. Lambert, J. H. Jefferson, and A. M. Bratkovsky, Phys. Rev. B 59, 11936 (1999).

${ }^{41}$ S. Datta, Electronic Transport in Mesoscopic Systems (Cambridge University Press, Cambridge, England, 1995).

${ }^{42}$ B. G. Cook, P. Dignard, and K. Varga, Phys. Rev. B 83, 205105 (2011).

${ }^{43}$ Y. X. Xing, B. Wang, and J. Wang, Phys. Rev. B 82, 205112 (2010).

${ }^{44}$ H. Haug and A.-P. Jauho, Quantum Kinetics in Transport and Optics of Semiconductors (Springer, Berlin, 1998).

${ }^{45}$ A.-P. Jauho, N. S. Wingreen, and Y. Meir, Phys. Rev. B 50, 5528 (1994).

${ }^{46}$ D. Waldron, P. Haney, B. Larade, A. MacDonald, and H. Guo, Phys. Rev. Lett. 96, 166804 (2006).

${ }^{47}$ D. Waldron, V. Timoshevskii, Y. Hu, K. Xia, and H. Guo, Phys. Rev. Lett. 97, 226802 (2006).

${ }^{48}$ N. Troullier and J. L. Martins, Phys. Rev. B 43, 1993 (1991). 